\title{
Use of calculators with computer algebra systems in test assessment in engineering mathematics
}

\author{
V. Weiss ${ }^{1} \quad$ P. Tobin ${ }^{2}$ \\ (Received 8 January 2016; revised 26 May 2016)
}

\begin{abstract}
In recent years the number of engineering departments in Australia that permit use of CAS calculators in test assessments has been decreasing, although in some cases students are expected to use CAS computer packages such as Matlab or Mathematica for some assignment or project work. We examine the common arguments given by departments and academics who oppose use of CAS calculators in tests and examinations and contrast these with possible benefits of allowing students to use CAS calculators in tests and examinations in engineering mathematics subjects. We present examples to illustrate different types of examination questions that can be asked in a calculus exam for undergraduate engineering students where CAS calculators are permitted.
\end{abstract}

http://journal.austms.org.au/ojs/index.php/ANZIAMJ/article/view/10445 gives this article, (C) Austral. Mathematical Soc. 2016. Published June 8, 2016, as part of the Proceedings of the 12th Biennial Engineering Mathematics and Applications Conference. ISSN 1446-8735. (Print two pages per sheet of paper.) Copies of this article must not be made otherwise available on the internet; instead link directly to this URL for this article. 


\section{Contents}

1 Introduction

C52

2 Issues in use of CAS calculators

C53

3 CAS and data storage

C54

4 Time used in mastering technology

C55

5 Conceptual and procedural learning

C56

6 Discussion

C63

References

C63

\section{Introduction}

Computer algebra systems (CAS) have a long history of use in mathematics (more than three decades to date). Handheld CAS calculators have been used extensively at school level, in both classes and exam assessments, for at least 15 years. There are many different types of CAS, including computer packages such as Matlab, Maple, Mathematica and Wolfram Alpha, and hand-held calculators such as the TI-Nspire and Casio Classpad. CAS calculators are most suited to basic manipulation, equation solving at a basic level and techniques in calculus including antidifferentiation and differential equations. CAS calculators can solve most of the algebra and calculus required in a typical first-year or second-year service mathematics subject, such as engineering mathematics. CAS calculators are the most feasible type of CAS for use in most test and exam assessments due to their portability and reduced storage capability compared to computers, although, in certain specially designed exams, CAS on computers can also be appropriate [4, e.g.]. 
In Victoria and Western Australia a CAS calculator is expected in senior secondary school mathematics exams, and has been for a number of years, yet CAS use in exams in Australia at tertiary level is far less widespread and in recent years has been contracting. For example, engineering departments at Edith Cowan University in 2010 and Swinburne University in late 2011 banned CAS calculators in tests and exams across most units in their departments. Staff teaching mathematics at Edith Cowan University managed to modify the ban to obtain exemptions for linear algebra questions, but for other topics, such as calculus where CAS can have a big impact, these bans held firm. Even at universities where CAS is not outright banned in mathematics exams, it is common for a large number of mathematics teaching staff to have a negative attitude towards CAS for exam assessments and to ignore CAS when teaching. While there is no official ban on CAS calculators at Victoria University, most units are designed and taught with no reference to CAS, as use of such calculators is considered by many staff to not be "real mathematics" and to be just "pushing buttons" [5].

\section{Issues in use of CAS calculators}

It is neccessary to identify the common arguments against CAS in mathematics exams at university level. These include practical concerns about:

1. the possibility of cheating in exams due to the capabilities of CAS technology (Section 3);

2. time factors relating to how long it will take students to master the technology during their study of a unit (Section 4);

3. the impact CAS has on students' cognitive development in mathematics and perceived limitations to the type of knowledge than can be tested if CAS is permitted in exams (Section 5).

We will now examine each of these issues in turn. 


\section{CAS and data storage}

First, we consider the issue of CAS technology providing opportunities for students to cheat in exams. The bans imposed by engineering departments at Swinburne University and Edith Cowan University on CAS in exams were adopted, at least ostensibly, because of concerns about the data storage capabilities of handheld CAS calculators and the potential for students to store examinable material on their CAS calculator and access it during an exam. Some staff also had concerns about the remote access capability of some advanced CAS calculators being used by students who elect to cheat in an examination. We were informed by the Victorian Curriculum and Assessment Authority ${ }^{1}$ that normal CAS calculators do not have remote access capabilities when used in isolation and and this issue is not a concern for them.

While concerns about the data storage capability of CAS calculators are certainly valid, there are ways around this problem. Furthermore, this issue would not be of equal concern in all units taught by engineering departments. For example, in some units, formula sheets are provided and/or students can bring in their own "cheat sheets". In such cases, the storage of additional information in the memory of a CAS calculator may not give students much of an advantage. Our experience has not shown calculator stored data to be a problem in mathematics exams, provided questions do not merely ask students for definitions or memorised information but rather ask for the implementation of procedures in problem solving. Data storage issues may not be of such concern if invigilators of exams in which CAS calculators are permitted are trained to check students' calculators during an exam to ensure there is no prohibited material stored in the memory - although this procedure needs further consideration. Some examination bodies, such as the Victorian Curriculum and Assessment Authority, regard this training as unnecessary, preferring to hold some exams with no calculators and others with questions which should make storage irrelevant.

${ }^{1}$ Victorian Curriculum and Assessment Authority http://www.vcaa.vic.edu.au/ 


\section{Time used in mastering technology}

A second argument used against CAS technology in mathematics courses is that the time taken for students to master CAS will have a negative impact on their learning. For example, while not an opponent of the technology, Jardine [3] was concerned that time spent teaching students how to use technology to solve mathematics problems is not time spent teaching students mathematics. It is certainly true that the available time for completing courses is always under pressure, and this problem tends to be especially severe in service courses such as engineering mathematics. However, Buteau et al. [2] argued that the reduced time available is actually one motivation to increase the use of CAS technology in tertiary courses. In a maths course, substantial time may be dedicated to repetitious step by step hand-calculations of countless variants of calculus techniques designed for an array of specific functions. This raises the question of whether it is better to use calculators and computers to do some of the repetitive procedural work and to focus more on concept development and application. Also, different types of CAS take different times to master - for example, while higher end computer packages such as Mathematica and Matlab have greater power and capabilities than handheld CAS calculators, they also take longer for students to learn, as they contain much more complicated syntax compared to the relatively simple, menu-driven layout of most handheld CAS calculators. Therefore, at times handheld CAS calculators are more advantageous than CAS computer software, and vice versa. For instance, in an advanced mathematical modelling course a computer package such as Matlab, Mathematica or Maple is the most appropriate, for use in assignment work at least, while in most first-year engineering mathematics service subjects, a handheld CAS calculator would be sufficient, easiest to use in an exam setting and also quicker/easier for students to learn. 


\section{Conceptual and procedural learning}

The third argument commonly used against CAS technology in mathematics units is that it is adopted without proper consideration of its consequences for students' conceptual development. Risser [7] gives some examples of academics raising this concern in two American news magazines for professional mathematicians. This issue was also raised in public forums by some mathematicians at the Australian Mathematical Sciences Institute ${ }^{2}$, as well as by some academics in university departments. But this raises the question of whether traditional service maths courses focus more on procedural learning than conceptual learning, and certainly procedural learning seems to be tested more than conceptual learning [9]. Procedural learning is usually defined as that which "involves only memorizing operations with no understanding of underlying meanings" [1]. In contrast, "conceptual learning involves understanding and interpreting concepts and the relations between concepts" [1]. CAS calculators assist procedural learning most easily, which then makes more time available for conceptual learning. CAS calculators also allow multiple representations of solutions, including graphical solutions. Arslan [1] suggested that using technology to obtain multiple representations of solutions may assist in the teaching of concepts. Tall et al. [8] also addressed this issue in their survey on Technology and Calculus and suggested that using technology to explore concepts, while delaying procedural skills, could lead to improvements in conceptual learning.

To illustrate use of multiple-representation capabilities of a CAS calculator consider the following calculus problem.

Example 1. Solve the differential equation $y^{\prime}=\frac{1}{2}\left(1+y^{2}\right)$ such that $y(0)=$ -1 and graph the local solutions using direction fields.

Figure 1 shows the "deSolve" command on a TI-Nspire CAS calculator and its algebraic solution to Example 1. The solution is not expressed in the usual form $y=f(x)$, so, if the solution was required in this usual form, then a

${ }^{2}$ Australian Mathematical Sciences Institute http://amsi.org.au/ 
Figure 1: CAS solution of differential equation in Example 1.

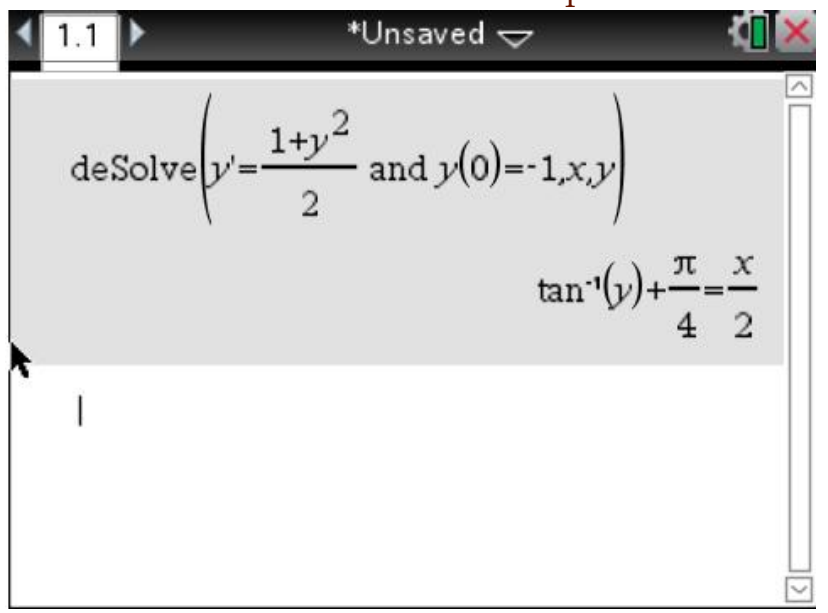

student would need to carry out at least one additional step, either by hand or on the calculator.

Figure 2 shows the graph of local solutions using direction fields, generated by the TI-Nspire for Example 1. Understanding the link between the analytic and graphical solutions and the DE is an example where CAS can assist with conceptual learning.

Effective use of CAS to help conceptual learning needs careful thought by teachers and course designers. But will it be effective if CAS is just grafted onto the existing classical course, or does the whole unit need rethinking? Most tertiary institutions will not allow radical redesign of a course to incorporate CAS (they would see it as a dangerous experiment, despite the limitations of the present courses).

The last common concern about CAS in exam assessment that we discuss is that of the perceived limitations in the type of knowledge that can be tested in exams where CAS is permitted - that is, exam questions will be "too easy" and in depth knowledge of the subject matter will not be tested. It is 
Figure 2: CAS graphical solution of differential equation in Example 1.

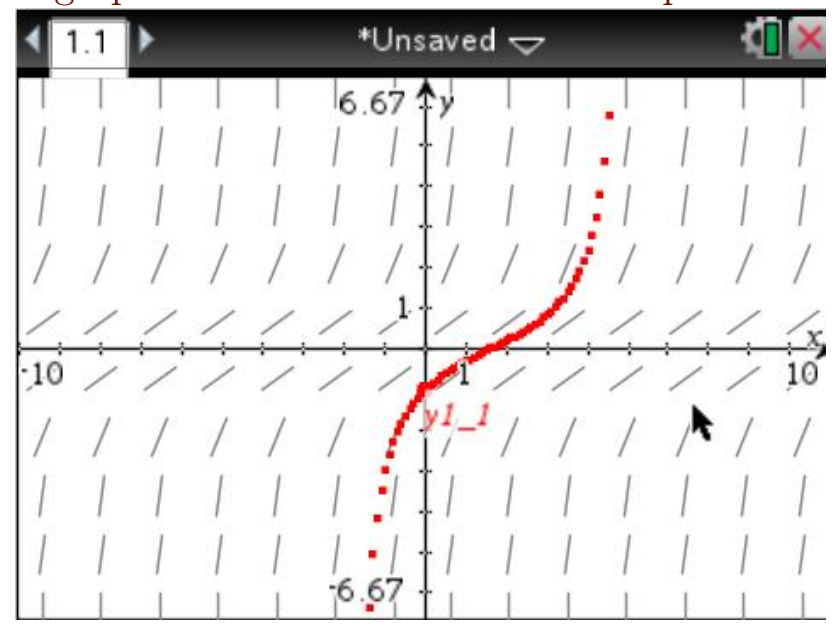

certainly true that exam questions can merely involve "pushing buttons" if they are not specifically designed with CAS in mind. For example, consider the following question from a 2008 first-year engineering mathematics exam paper.

Example 2. Solve the DE $\mathbf{y}^{\prime \prime}-4 y=e^{2 x}$.

The question in Example 2 does not test procedural knowledge of the steps involved in solving the DE, nor does it test any conceptual knowledge - it only tests whether a student knows how to use the correct commands/menus on the CAS calculator. In itself, that could be a useful skill to test if procedural and conceptual knowledge skills are also tested in the exam, but a question like Example 2 should not be worth many marks if the students have access to CAS calculators.

A question directly testing calculator skills, without requiring any demonstration of intermediate steps, could be useful, provided the assessment task also includes a more complicated question involving an application of the technique being tested and/or a more complicated mathematical expression. With this 
in mind, consider the following example, which is based on a question that was asked in a past mid-unit test paper.

Example 3. Air temperature $\mathrm{T}$ in a particular area depends on time of day $\mathrm{t}$ and the elevation above sea level $z$, with $T=T_{0}[1+\cos (\pi t / 12)] e^{-k z}$, where $T_{0}$ and $k$ are constants. A hot air balloon rises vertically. The balloon's altitude at time $t$ is given by $z=\sin (v t)$, where $v$ is a constant. Determine the rate of temperature variation observed by a balloon passenger.

There are various methods students could use to solve Example 3. If they try to get the answer in one step by replacing $z$ with $\sin (\nu \mathrm{t})$, then some calculators, including the TI-Nspire, will give the answer immediately. However, a Casio Classpad model we used gave the output

$$
-\left(k v \cos (v t) T_{0}[\cos (\pi t / 12)+1]-\frac{d}{d t} T_{0}[\cos (\pi t / 12)+1]\right) e^{-k \sin (v t)} .
$$

In order to complete the derivative, students need to understand that they are differentiating $z$ with respect to $t$, while $k, T_{0}$ and $v$ are constants. A similar idea could be applied to a differential equations question or other calculus questions in an applied context.

Questions similar to Example 3 can also be useful if the students are asked to show that their answer is equivalent to a different form of the solution, or to choose the correct answer from a selection of calculator outputs that initially appear different to the answer given by their CAS calculator, thus requiring them to understand different algebraic representations of the same solution.

Now consider a question designed to test students' procedural knowledge of the steps involved in solving a second-order linear differential equation with constant coefficients.

Example 4. Consider the differential equation $y^{\prime \prime}-4 y=e^{2 x}$.

(a) Write the auxiliary equation for this differential equation.

(b) Determine the complementary function for this differential equation. 
(c) Determine a particular integral for this differential equation. Show all working.

(d) Write the general solution of this differential equation.

Any student who does not understand the process at all but knows how to use CAS to solve such a DE would write an answer for part (d) only. Students who do not know the full process but who know that the solution is the complementary function plus the particular integral might use the CAS to get the general solution and then write down answers for (b) and (c) as well, but without showing any working and without necessarily giving any answer for part (a). Asking Example 4 in this way limits the students' use of CAS. If students need to know all these parts (as is common in extant classical service engineering maths courses), then this question allows the knowledge to be reinforced with CAS technology. But, students who rely on CAS to solve the $\mathrm{DE}$ are required to reflect on the structure of the solution produced by their CAS calculator in order to get more than part (d) of the question correct.

Exam questions that provide students with CAS calculator output and require them to interpret it or use it in solving a related problem are often useful. Consider the following exam question.

Example 5. The differential equation $y^{\prime \prime}-9 y=e^{3 x}$ was inputted into a CAS calculator and generated the output shown in Figure 3. From this output, identify a suitable particular integral independent of the complementary function.

Example 5 gives students the general solution of the differential equation, but the question tests students' understanding of the components of the CAS output by testing if they can identify the particular integral and complementary function in the general solution.

The solution in Figure 3 generated by the calculator is in a slightly different form to that which is obtained by the method of undetermined coefficients, commonly taught in first-year maths courses for solving DEs such as that in Example 5. The CAS writes the solution as $y=\left(\frac{x}{6}+c_{2}-\frac{1}{36}\right) e^{3 x}+c_{1} e^{-3 x}$ 
Figure 3: CAS solution to differential equation in Example 5.

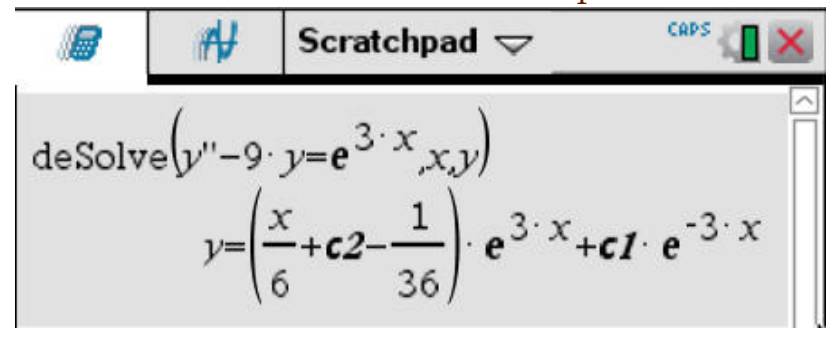

(Figure 3), while the solution usually obtained by hand is $y=c_{1} e^{-3 x}+c_{2} e^{3 x}+$ $\frac{x}{6} e^{3 x}$. Therefore, this question requires students to reflect on the output and understand, for instance, how the constants are represented differently in these two solutions but are essentially giving the same solution to the problem. When using CAS to solve problems involving integration of circular functions, there often is even more variety in outputs, as discussed by PonceCampuzano and Rivera-Figueroa [6]. Furthermore, some CAS packages give a trigonometric solution that is inconsistent with the domain over which the integral is defined [6], which is important to be aware of when writing exams for students with CAS calculators. Many calculators such as the TI-Nspire give warnings about domain issues when solving problems.

Some exam questions involving CAS calculator output are also useful in testing conceptual understanding. For example, consider this question from a past exam paper in a first-year maths unit.

Example 6. The slope field for the DE $y^{\prime}=-0.25(y-1)(y-5)$ is shown in Figure 4. Axes are solid lines with $y$ the vertical axis. Identify the initial condition which generates the particular solution curve shown in red and explain the local slopes shown in terms of the DE.

Slope fields give us some understanding of DE solutions under various initial conditions and the output shown in Figure 4 also includes the curve (red) for a particular solution determined by a specific initial condition. Horizontal slopes in Figure 4 must occur when $y^{\prime}=0$ and the right-hand side of the DE 
Figure 4: Slope field of differential equation in Example 6.

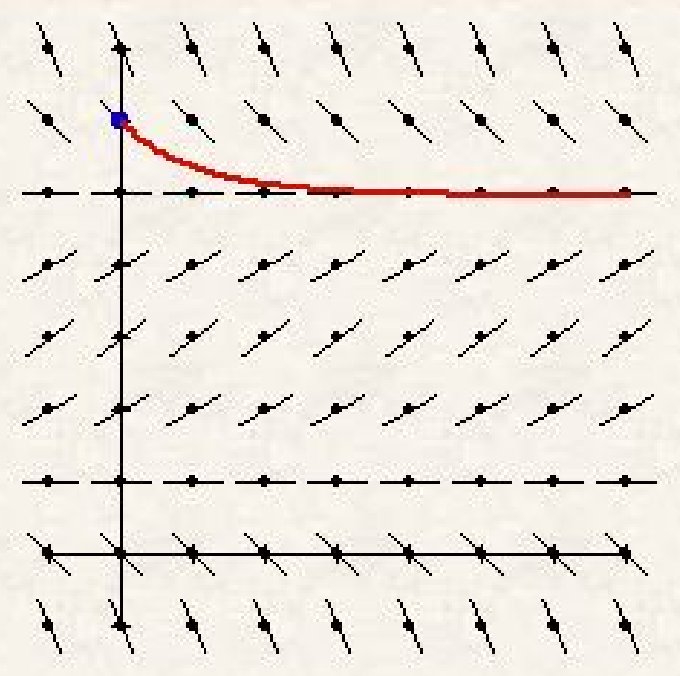

shows that this occurs when $y=1,5$. Students are expected to identify that the horizontal line segments in Figure 4 indicate $y=1$ and $y=5$ and hence determine that the initial condition of the red curve is $y(0)=6$. Therefore, students need some conceptual understanding of how the graphical output relates to the solution of the $\mathrm{DE}$.

Student attempts at this question were overall quite poor, suggesting more focus is needed on interpreting such graphical representations of DE solutions. It would also be worth investigating why students have difficulty interpreting this type of computer output.

The exam questions discussed above in Examples 1-6 are from the field of calculus and demonstrate that exam questions involving CAS can test a lot more than merely whether students know the correct menus to select/buttons to press. A wide range of CAS suitable exam questions could also be asked when examining other topic areas. 


\section{Discussion}

The extent to which CAS can be used effectively is still not resolved. The arguments mounted against its use in test situations seem to be flawed, at least in part. The increasing pressure on teaching time for service courses, such as engineering mathematics, makes it desirable to find ways to teach more efficiently. The problem then remaining is whether there is a serious impact on conceptual learning and whether the tools allow more options for cheating. What CAS can do for calculus and algebra is similar to what statistical packages can do for correlations, analysis of variance, descriptive statistics, etc. It is much more useful for students to know how to use packages to solve problems and interpret solutions in statistics - shouldn't we do the same in mathematics?

\section{References}

[1] S. Arslan. Traditional instruction of differential equations and conceptual learning. Teach. Math. Appl. 29(2):94-107, 2010. doi:10.1093/teamat/hrq001 C56

[2] C. Buteau, N. Marshall, D. Jarvis and Z. Lavicza. Integrating computer algebra systems in post-secondary mathematics education: Preliminary results of a literature review. Int. J. Tech. Math. Ed. 17(2):57-68, 2010. https://www. learntechlib.org/p/107456 C55

[3] D. Jardine. A different pencil: Use technology wisely. MAA Focus, 21(6):20-21, 2001. http:

//www. maa.org/sites/default/files/pdf/pubs/augsept01web.pdf C55

[4] H. Lehning. Exams and computer algebra systems. Proceedings of the Second International Conference on the Teaching of Mathematics, 
Hersonissos, Crete, Greece, 1-6 July, 2002.

http://www.math.uoc.gr/ ictm2/Proceedings/pap40.pdf C52

[5] A. McAndrew. Private communication. C53

[6] J. C. Ponce-Campuzano and A. Rivera-Figueroa. Using computer algebraic systems to compute antiderivatives: Showing some mathematical facts that should not be neglected. In J. Hannah and M. Thomas (Eds), Proceedings of Volcanic Delta 2011, the Eighth Southern Hemisphere Conference on Teaching and Learning Undergraduate Mathematics and Statistics, Rotarua NZ, 27 Nov.-2 Dec., 303-313, 2011. http://www. math. canterbury.ac.nz/ j.hannah/Delta11/ VolcanicDelta2011Proceedings.pdf C61

[7] H. S. Risser. What are we afraid of? Arguments against teaching mathematics with technology in the professional publications of organisations for US mathematicians Int. J. Tech. Math. Ed. 18(2):97-101, 2011. https://www. learntechlib.org/p/109511 C56

[8] D. Tall, D. Smith and C. Piez. Technology and calculus. In M. K. Heid and G. W. Blume (Eds), Research on Technology and the Teaching and Learning of Mathematics, Vol 1, 207-258. NCTM, 2008.

https://www.nctm.org/store/Products/

Research-on-Technology-and-the-Teaching-and-Learning-of-Mathem -Research-Syntheses/ C56

[9] P. Tobin and V. Weiss. Teaching differential equations in undergraduate mathematics: Technology issues in service courses. In J. Hannah and M. Thomas (Eds), Proceedings of Volcanic Delta 2011, the Eighth Southern Hemisphere Conference on Teaching and Learning Undergraduate Mathematics and Statistics, Rotarua NZ, 27 Nov.-2 Dec., 375-385, 2011. http://www. math. canterbury.ac.nz/ j.hannah/Delta11/ VolcanicDelta2011Proceedings.pdf C56 


\section{Author addresses}

1. V. Weiss, Swinburne University and Australian Catholic University, Melbourne, Australia mailto:vweiss@swin. edu . au

2. P. Tobin, Australian Catholic University, Melbourne, Australia mailto:patrick.tobin@acu.edu.au 